fibre is the $n$-dimensional complex projective space (complex projective co-tangent bundle). This bundle of complex dimension $2 n+1$ is our $M$. Considering the fibre of $T(V)$ as the $(2 n+2)$-dimensional real vector space, we take as $P$ the co-tangent sphere bundle over $V$ (i.e., the fibre of $P$ is a sphere in the fibre of $\widetilde{T}(V)$ ).

$\tilde{T}(V)-V$ is the principal fibre bundle associated with a line bundle $L$ over $M$. The definition of the complex contact structure on $M$ is similar to the one in the first example. The classical real contact structure on the co-tangent sphere bundle $P$ is the one derived from the complex contact structure on the complex projective co-tangent bundle $M$ as described in the proof of (3).

\title{
BIBLIOGRAPHY
}

1. A. Borel and F. Hirzebruch, Characteristic classes and homogeneous spaces, I, Amer. J. Math. vol. 80 (1958) pp. 458-538.

2. S. S. Chern, Pseudo-groupes continus infinis, Colloque de Geométrie Diff., Strasbourg 1953.

3. C. Chevalley, Theory of Lie groups I, Princeton, 1946.

4. J. W. Gray, Some global properties of contact structures, to appear.

Institute for Advanced Study

\section{FIXED POINTS FOR MULTI-VALUED FUNCTIONS ON SNAKE-LIKE CONTINUA}

\author{
RONALD H. ROSEN ${ }^{1}$
}

1. Introduction. A multi-valued function from a space $X$ into a space $Y$ is a point to set correspondence. Hamilton has shown that snake-like continua have the fixed point property with respect to maps [2]. Ward has extended this to show that snake-like continua have the fixed point property with respect to continuous multivalued functions [6]. The results of this paper establish that a more general class of spaces, those which are inverse limits of arcs, have even stronger properties with respect to multi-valued functions. ${ }^{2}$

2. Multi-valued functions. All functions are multi-valued unless otherwise indicated. A map will always be a continuous single-valued function.

Presented to the Society, April 19, 1958; received by the editors June 5, 1958.

1 National Science Foundation Fellow.

2 The author wishes to thank Professor E. R. Fadell for his encouragement and for reading and criticizing this paper. 
Definitions. For a space $Y$, let $K(Y)$ denote the class of nonempty subsets of $Y$. A function $f: X \rightarrow K(Y)$ will be called a multivalued function of $X$ into $Y$. For each $x$ in $X, f(x)$ is a subset of $Y$. For $A \subseteq X$, let $f(A)=\bigcup_{x \in A} f(x)$. $f$ will be called onto if $f(X)=Y$. If $f: X \rightarrow Y$ and $g: Y \rightarrow Z$, then the composition of $f$ and $g$ is $g f: X \rightarrow Z$, where for each $x$ in $X, g f(x)=g(f(x))$. Associated with each function $f$ is another function $f^{-1}$ defined on $f(X)$. If $y$ is in $Y, f^{-1}(y)=\{x \mid f(x) \ni y\}$ (if $y \notin f(X), f^{-1}(y)=0$ ); if

$$
B \subseteq Y, f^{-1}(B)=\bigcup_{\boldsymbol{y} \in B} f^{-1}(y)=\{x \mid f(x) \cap B \neq 0\} .
$$

Note that $\left(f^{-1}\right)^{-1}=f$ for any function $f$. A function $f$ will be called point closed, if $f(x)$ is closed for each point $x$ in $X$; point connected, if $f(x)$ is connected for each $x$ in $X$; and closed (open) if the image under $f$ of every closed (open) set in $X$, is closed (open) in $Y$.

$f: X \rightarrow Y$ is upper-semi-continuous (u.s.c.) if it is point closed and $f^{-1}$ is closed; $f$ is lower-semi-continuous (l.s.c.) if $f^{-1}$ is open; finally, $f$ is continuous if it is both u.s.c. and l.s.c. It is immediate for compact (the finite covering property) Hausdorff spaces, that the composition of two u.s.c (1.s.c.) functions is u.s.c. (1.s.c.).

Let $A$ and $A^{\prime}$ denote our definitions of u.s.c. and 1.s.c. We also note the following conditions:

B. For each point $x$ in $X$ and open set $U$ in $Y$ such that $f(x) \subseteq U$, there is a neighborhood $N$ of $x$ so that $x^{\prime} \in N$ implies $f\left(x^{\prime}\right) \subseteq U$.

C. For any converging sequence of points in $X$,

$$
\left(x_{n}\right) \rightarrow x_{0}, \lim _{n} \sup f\left(x_{n}\right) \subseteq f\left(x_{0}\right) .
$$

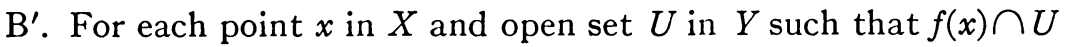
$\neq 0$, there is a neighborhood $N$ of $x$ so that if $x^{\prime} \in N, f\left(x^{\prime}\right) \cap U \neq 0$.

$\mathrm{C}^{\prime}$. For any converging sequence of points in $X$,

$$
\left(x_{n}\right) \rightarrow x_{0}, f\left(x_{0}\right) \subseteq \lim _{n} \inf f\left(x_{n}\right) .
$$

In general, for point closed functions $f, A$ implies $B$ and $B$ implies $C$; if $X$ is metric and $Y$ compact Hausdorff, $A, B$, and $C$ are equivalent. For all functions $f, A^{\prime}$ and $B^{\prime}$ are equivalent and both imply $C^{\prime}$; if $X$ is metric, all three are equivalent. $C$ and $C^{\prime}$ are the definitions used by Strother [5].

If $f: X \rightarrow Y, G(f)$, the graph of $f$, is the set $\{(x, y) \mid y \in f(x)\}$ contained in $X \times Y$, the cartesian product of $X$ and $Y$. When $f$ and $g$ are both functions of $X$ into $Y, g>f$ ( $g$ refines $f$ ) if for each $x \in X, g(x)$ $\subseteq f(x)$. 
Henceforth all spaces are assumed to be compact Hausdorff.

Lemma 1. Let $f: X \rightarrow Y$ be continuous and $X$ be connected. There is an u.s.c. function $g: X \rightarrow Y$ such that $g>f$ and the graph of $g$ is con: nected.

Proof. Let $\tilde{f}: X \rightarrow G(f)$ be defined as follows, $\tilde{f}(x)=(x, f(x))$. $\tilde{f}$ is continuous and onto. By Lemma 3 of [4], if $B$ is a component of $G(f), \tilde{f}^{-1}(B)=X$. Next we define $\tilde{g}: X \rightarrow B, \tilde{g}(x)=\tilde{f}(x) \cap B$. Denote by $\pi$ the natural projection of $X \times Y$ onto $Y . \tilde{g}$ is clearly point closed. If $A$ is a closed subset of $B, \tilde{g}^{-1}(A)=\{x \mid \tilde{g}(x) \cap A \neq 0\}=\tilde{f}^{-1}(A)$. It follows that $\tilde{g}$ is u.s.c. and so $g=\pi \tilde{g}: X \rightarrow Y$ is also u.s.c. For each $x \in X$, $g(x)=\pi \tilde{g}(x)=\pi(\tilde{f}(x) \cap B)=\pi((x, f(x)) \cap B) \subseteq f(x)$. It is easily seen that $B=G(g)$.

Lemma 2. Let $X, Y, Z$, and $W$ be spaces, $f: Y \rightarrow X$ a map onto, $g: Y \rightarrow Z$ u.s.c., $h: Z \rightarrow W$ a map, and $G(g)$ connected. Then $G\left(h g f^{-1}\right)$ is connected.

PRoof. Let $\pi_{1}, \pi_{2}$, and $\pi_{3}$ be the natural projections of $X \times Y \times Z$ onto $X \times Y, Y \times Z$, and $X \times Z$ respectively. Define

$$
\mathcal{S}=\left\{(x, y, z) \mid y \in f^{-1}(x) \& z \in g(y)\right\} \subseteq X \times Y \times Z .
$$

We note that $\pi_{1}(\mathcal{G})=G\left(f^{-1}\right), \pi_{2}(\mathcal{G})=G(g)$, and $\pi_{3}(\mathcal{G})=G\left(g f^{-1}\right)$.

$g$ is closed as may be seen by a straight-forward application of the u.s. continuity of $f^{-1}$ and $g$. $G$ is also connected. If we deny this, there is a partition $\mathcal{G}=A / B$. As $G(g)$ is connected, there is a point $(y, z)$ $\in \pi_{2}(A) \cap \pi_{2}(B) \subseteq G(g)$. Then there must exist points $x_{1}, x_{2}$ in $X$ so that $\left(x_{1}, y, z\right) \in A$ and $\left(x_{2}, y, z\right) \in B$. Therefore since $f$ is a map, $f(y)=x_{1}=x_{2}$, a contradiction. Define $\hat{h}: G\left(g f^{-1}\right) \rightarrow G\left(h g f^{-1}\right)$ by $\hat{h}(x, z)$ $=(x, h(z))$. As $h$ is a map, $\hat{h}$ is a map onto and $G\left(h g f^{-1}\right)$ is connected.

If $f$ and $g$ are both functions from $X$ into $Y$ and there is a point $x$ in $X$ so that $f(x) \cap g(x) \neq 0, x$ will be called an incidence point of $f$ and $g$; if $X=Y$ and $g$ is the identity map, $x$ will be called a fixed point of $f$.

Lemma 3. Suppose $f$ and $g$ are two functions from an arc $I$ into an arc $I^{\prime}, f$ is u.s.c. and onto, and $G(f)$ and $G(g)$ are both connected. Then $f$ and $g$ have an incidence point.

Proof. We shall show that $G(f) \cap G(g) \neq 0 . I \times I^{\prime}$ is homeomorphic to the unit square $I^{2}=\{(x, y) \mid 0 \leqq x \leqq 1,0 \leqq y \leqq 1\} \subseteq E^{2}$. We assume $I$ $=\{(x, y) / y=0\} \subseteq I^{2}$ and $I^{\prime}=\{(x, y) / x=0\} \subseteq I^{2}$. As $f$ is onto, $G(f)$ intersects the horizontal sides of $I^{2},\{(x, y) \mid y=0\}$ and $\{(x, y) \mid y=1\}$. If we assume $G(f) \cap G(g)=0$, then $(G(g) \cap\{(x, y) \mid x=0\})$ and 
$(G(g) \cap\{(x, y) \mid x=1\})$ are nonempty subsets of $G(g)$ which are separated by the continuum $G(f)$, contradicting the connectedness of $G(g)$.

3. Inverse limits and snake-like continua. We shall use inverse systems as defined in [1].

$$
X=\lim _{\leftarrow}\left\{X_{\alpha}, \pi_{\alpha}^{\beta}\right\}_{\alpha, \beta \in A}
$$

will mean that $X$ is the inverse limit of the inverse system $\left\{X_{\alpha}, \pi_{\alpha}^{\beta}\right\}_{\alpha, \beta \in A}$ where $\left\{\pi_{\alpha}^{\beta}\right\}_{\alpha, \beta \in A}$ is the class of projection maps and $A$ is the directed set. We recall that the inverse limit of compact nonempty Hausdorff spaces is again a compact nonempty Hausdorff space. If $A$ and $A^{\prime}$ are two directed sets, a function $\phi: A \rightarrow A^{\prime}$ will be called a homomorphism if $\alpha \leqq \beta$ in $A$ implies that $\phi(\alpha) \leqq \phi(\beta)$ in $A^{\prime}$ and $\phi(A)$ is cofinal in $A^{\prime}$; a one-to-one homomorphism will be called an isomorphism.

A chain is a finite collection of open sets $\left(u_{1}, \cdots, u_{k}\right)$ so that $u_{i} \cap u_{j} \neq 0$ if and only if $|i-j| \leqq 1, i, j=1, \cdots, k$. A compact continuum will be called chainable if each of its open coverings has a refinement which is a chain. A compact metric chainable continuum is called a snake-like continuum. It is known that each snake-like continuum $S$ is the inverse limit of a sequence of arcs (see [3] for an even more general result); if $S$ is nondegenerate we may assume that all the projection maps are onto.

It is not hard to prove that if $X$ is the inverse limit of arcs, it is a chainable continuum. It would be interesting to know if each compact chainable Hausdorff continuum is the inverse limit of arcs. Here are two examples of nonmetric compact continua which are the inverse limit of arcs.

(1) Let $[1, \Omega]$ be all the ordinals from 1 to $\Omega$, the first ordinal preceded by an uncountable number of ordinals. Let $X$ be the space constructed from $[1, \Omega]$ by connecting each ordinal and its suctessor (save, of course, $\Omega$ ) by an open arc so that each such pair of distinct arcs are disjoint. $X$ has a natural order topology preserving limit points in the ordinal sense.

(2) Construct $Y$ from $[1, \Omega]$ using pseudo-arcs instead of arcs. $Y$ is not ordered, but limit points in the ordinal sense are to be preserved in its topology.

The directed set for both $X$ and $Y$ may be chosen to be the set of all ordinals less than $\Omega$.

THEOREM 1. Let $X$ and $Y$ be the inverse limits of inverse systems of arcs over directed sets $A$ and $A^{\prime}$, respectively, and $\phi$ an isomorphism of $A$ into $A^{\prime}$. Suppose $f$ and $g$ are functions of $X$ into $Y$, $f$ and $g$ are u.s.c., 
$f$ is onto, and $G(f)$ and $G(g)$ are both connected. Then $f$ and $g$ have an incidence point.

Proof. Let

$$
X=\lim _{\leftarrow}\left\{I_{\alpha}, \pi_{\alpha}^{\beta}\right\}_{\alpha, \beta \in A}, \quad Y=\lim _{\leftarrow}\left\{I_{\alpha^{\prime}}, \stackrel{\beta_{\alpha^{\prime}}^{\beta^{\prime}}}{\beta_{\alpha^{\prime}, \beta^{\prime} \in A^{\prime}}}\right.
$$

We assume $\pi_{\alpha}^{\beta}, \rho_{\alpha^{\prime}}^{\beta}$ are onto for each $\alpha, \beta, \alpha^{\prime}, \beta^{\prime}$, since the cases when either $X$ or $Y$ is degenerate are trivial. Define $f_{\alpha}=\rho_{\phi(\alpha)} f \pi_{\alpha}^{-1}: I_{\alpha} \rightarrow I_{\phi(\alpha)}$ and $g_{\alpha}=\rho_{\phi(\alpha)} g \pi_{\alpha}^{-1}: I_{\alpha} \rightarrow I_{\phi(\alpha)}$ for each $\alpha$ in $A$. Each $f_{\alpha}, g_{\alpha}$ is u.s.c. By Lemma 2, $G\left(f_{\alpha}\right)$ and $G\left(g_{\alpha}\right)$ are connected. Since $\pi_{\alpha}^{-1}, f$, and $\rho_{\phi(\alpha)}$ are onto, $f_{\alpha}$ is onto. By Lemma 3, as $I_{\alpha}$ and $I_{\phi(\alpha)}$ are arcs, $f_{\alpha}$ and $g_{\alpha}$ have an incidence point.

For each $x$ in $X$, define

$$
F(x)=\lim _{\leftarrow}\left\{f_{\alpha} \pi_{\alpha}(x), \stackrel{\phi(\beta)}{\rho_{\phi(\alpha)}}\right\}
$$

and $G(x)$ similarly with respect to the functions $g_{\alpha} \pi_{\alpha}(x)$. We shall show that $f(x)=F(x)$ and $g(x)=G(x)$.

For each $\alpha \leqq \beta, \rho_{\phi(\alpha)}^{\phi(\beta)} f_{\beta}>f_{\alpha} \pi_{\alpha}^{\beta}$. Let $\xi \in I_{\beta}$. Then $\pi_{\alpha}^{\beta-1} \pi_{\alpha}^{\beta}(\xi) \ni \xi$, so $\pi_{\alpha}^{-1} \pi_{\alpha}^{\beta}(\xi) \supseteq \pi_{\beta}^{-1}(\xi)$. Hence $f_{\alpha} \pi_{\alpha}^{\beta}(\xi) \supseteq \rho_{\phi(\alpha)} f \pi_{\beta}^{-1}(\xi)=\rho_{\phi(\alpha)}^{\phi(\beta)} f_{\beta}(\xi)$. In particular, then, $\rho_{\phi(\alpha)}^{\phi(\beta)} f_{\beta} \pi_{\beta}(x) \subseteq f_{\alpha} \pi_{\alpha}(x)$. This means that $F(x)$ is well-defined.

For each $\alpha, \rho_{\phi(\alpha)} f(x) \subseteq \rho_{\phi(\alpha)} f \pi_{\alpha}^{-1}\left(\pi_{\alpha}(x)\right)=f_{\alpha} \pi_{\alpha}(x)$, so $f(x) \subseteq F(x)$.

Suppose $y \in Y-f(x)$. Using the fact that $W=\left\{\pi_{\alpha}^{-1}(U)\right\}, W^{\prime}$ $=\left\{\rho_{\phi(\alpha)}^{-1}(V)\right\}$ are bases respectively for $X$ and $Y$, where $\alpha$ takes on all values in $A, U$ runs over all open sets in $I_{\alpha}$, and $V$, over all open sets in $I_{\phi(\alpha)}$; there is an $\alpha \in A$ and open sets $V$ and $V^{\prime}$ in $I_{\phi(\alpha)}$ such that $\rho_{\phi(\alpha)} f(x) \subseteq V, \rho_{\phi(\alpha)}(y) \in V^{\prime}$, and $V \cap V^{\prime}=0$. Since $f$ is u.s.c., there is a neighborhood $N$ of $x$ so that $f(N) \subseteq \rho_{\phi(\alpha)}^{-1}(V), N=\pi_{\beta}^{-1}(U), \beta>\alpha$, and $U$ is open in $I_{\beta}$. If $\xi \in U, f \pi_{\beta}^{-1}(\xi) \subseteq \rho_{\phi(\alpha)}^{-1}(V)$. Therefore $f_{\beta}(\xi)$ $\subseteq \rho_{\phi(\beta)} \rho_{\phi(\alpha)}^{-1}(V)=\rho_{\phi(\alpha)}^{\phi(\beta)}-1(V), \rho_{\phi(\beta)}(y) \in \rho_{\phi(\alpha)}^{\phi(\beta)}\left(V^{\prime}\right)$, and

$$
\stackrel{\phi(\beta)-1}{\rho_{\phi(\alpha)}}(V) \cap \stackrel{\phi(\beta)-1}{\rho_{\phi(\alpha)}}\left(V^{\prime}\right)=0 .
$$

Thus $\rho_{\phi(\beta)}(y) \notin f_{\beta} \pi_{\beta}(x) \subseteq \rho_{\phi(\alpha)}^{\phi(\beta)}(V)$, implying that $y \notin F(x)$. The proof for $g(x)$ is exactly the same.

For each $\alpha$, let $P_{\alpha}$ be the set of incidence points for $f_{\alpha}$ and $g_{\alpha}$. We have already seen that each $P_{\alpha}$ is nonempty. The sets $P_{\alpha}$ are closed. Suppose $\lambda \in I_{\alpha}-P_{\alpha}$ so that $f_{\alpha}(\lambda) \cap g_{\alpha}(\lambda)=0$. There are open sets $U$ and $V$ in $I_{\phi(\alpha)}$ so that $f_{\alpha}(\lambda) \subseteq U, g_{\alpha}(\lambda) \subseteq V$, and $U \cap V=0$. There is a neighborhood $W$ of $\lambda$ so that $f_{\alpha}(W) \subseteq U$ and $g_{\alpha}(W) \subseteq V$, hence $W \cap P_{\alpha}$ $=0$.

For $\alpha<\beta, \pi_{\alpha}^{\beta}\left(P_{\beta}\right) \subseteq P_{\alpha}$. Let $\xi \in P_{\beta}$. As was shown above, $\rho_{\phi(\alpha)}^{\phi(\beta)} f_{\beta}(\xi)$ 
$\subseteq f_{\alpha} \pi_{\alpha}^{\beta}(\xi)$ and similarly for $g_{\alpha}$ and $g_{\beta}$. This implies, $f_{\alpha} \pi_{\alpha}^{\beta}(\xi) \cap g_{\alpha} \pi_{\alpha}^{\beta}(\xi)$ $\supseteq \rho_{\phi(\alpha)}^{\phi(\beta)} f_{\beta}(\xi) \cap \rho_{\phi(\alpha)}^{\phi(\beta)} g_{\beta}(\xi) \supseteq \rho_{\phi(\alpha)}^{\phi(\beta)}\left(f_{\beta}(\xi) \cap g_{\beta}(\xi)\right) \neq 0$. Accordingly

$$
P=\lim _{\leftarrow}\left\{P_{\alpha}, \pi_{\alpha}^{\beta}\right\}
$$

is closed and nonempty.

$P$ is the set of incidence points for $f$ and $g$.

If $x \in P$, then for each $\alpha, f_{\alpha} \pi_{\alpha}(x) \cap g_{\alpha} \pi_{\alpha}(x) \neq 0$ and if $\alpha<\beta$, $\rho_{\phi(\alpha)}^{\phi(\beta)}\left(f_{\beta} \pi_{\beta}(x) \cap g_{\beta} \pi_{\beta}(x)\right) \subseteq f_{\alpha} \pi_{\alpha}(x) \cap g_{\alpha} \pi_{\alpha}(x)$. This means that

$$
L=\lim _{\leftarrow}\left\{f_{\alpha} \pi_{\alpha}(x) \cap g_{\alpha} \pi_{\alpha}(x), \stackrel{\phi(\beta)}{\rho_{\phi(\alpha)}}\right\} \neq 0
$$

and

$$
L \subseteq \lim _{\leftarrow}\left\{f_{\alpha} \pi_{\alpha}(x), \stackrel{\phi(\beta)}{\rho_{\phi(\alpha)}}\right\} \cap \lim _{\leftarrow}\left\{g_{\alpha} \pi_{\alpha}(x), \stackrel{\phi(\beta)}{\rho_{\phi(\alpha)}}\right\}=f(x) \cap g(x) .
$$

If $x \in X$ and $f(x) \cap g(x) \neq 0$, then for each $\alpha, f_{\alpha} \pi_{\alpha}(x) \cap g_{\alpha} \pi_{\alpha}(x)$ $\supseteq \rho_{\phi(\alpha)} f(x) \cap \rho_{\phi(\alpha)} g(x) \supseteq \rho_{\phi(\alpha)}(f(x) \cap g(x)) \neq 0$. Since $\pi_{\alpha}(x) \in P_{\alpha}, x$ is in $P$.

Corollary 1. Suppose $g: S \rightarrow S, S$ is a snake-like continuum, $g$ is u.s.c., and the graph of $g$ is connected. Then $g$ has a fixed point.

Corollary 2. Suppose $f$ and $g$ are maps from $S$ into $T$, two snakelike continua, and $f$ is onto. Then $f$ and $g$ have an incidence point.

Proof. The directed set for $S$ and $T$ may be taken to be the integers and $\phi$ the identity homomorphism. As is well known, the graph of a map defined on a continuum is connected.

Theorem 2. Suppose $X$ and $Y$ are as in Theorem 1. Let $f$ and $g$ be u.s.c. functions of $X$ into $Y, f$ onto, and $f$ and $g$ point connected. Then $f$ and $g$ have an incidence point.

Proof. This is an immediate consequence of Theorem 1 and the fact that a point connected u.s.c. function defined on a continuum has a connected graph.

Corollary. Let $g: S \rightarrow S, S$ be a snake-like continuum and $g$ u.s.c. and point connected. Then $g$ has a fixed point.

Theorem 3. Suppose $X$ and $Y$ are as in Theorem 1. Let $f$ and $g$ be functions of $X$ into $Y, f$ u.s.c. and onto, $G(f)$ connected, and $g$ continuous. Then $f$ and $g$ have an incidence point.

Proof. By Lemma 1, there is an u.s.c. function $g^{\prime}: X \rightarrow Y$ so that $g^{\prime}>g$ and $G\left(g^{\prime}\right)$ is connected. From Theorem 1, there is a point $x \in X$ for which $f(x) \cap g^{\prime}(x) \neq 0$. Since $g^{\prime}(x) \subseteq g(x), f(x) \cap g(x) \neq 0$. 
Corollary. Let $g: S \rightarrow S, S$ be a snake-like continuum and $g$ continuous. Then $g$ has a fixed point.

4. Remarks. Theorem 3 is false if we replace the conditions that $f$ is u.s.c. and $G(f)$ is connected by making $f$ continuous. In the unit square define $A, B$, and $C$ to be the respective line segments joining the pairs of points $(0,0)$ and $(1,1 / 2),(0,1 / 4)$ and $(1,3 / 4)$, and $(0,1 / 2)$ and $(1,1)$. Let $A \cup C$ be the graph of $f$ and $B$ the graph of $g$; $f$ and $g$ are functions from the bottom side of the square into the left side, both are continuous, and $f$ is onto.

The proof of the corollary to Theorem 3 as first given by Ward in [6] can be strengthened to show that a compact chainable Hausdorff continuum $X$ has a fixed point under any continuous multi-valued function of $X$ into itself, using the definitions $A$ and $A^{\prime}$ for continuity.

Corollary 2 to Theorem 1 may also be proved directly for compact chainable Hausdorff continua in a manner analogous to Hamilton's proof [2], using the following two lemmas.

Lemma A. Let $U$ and $T$ be finite linearly ordered sets. For an element $x$ in $U(T)$ let $\operatorname{Star} x$ be the set consisting of $x$, its predecessor, and its successor in the ordering of $U(T)$ (provided, of course, it has any). Suppose $f$ and $g$ are two maps of $U$ into $T$ so that $f$ is onto and for each $u \in U, f(\operatorname{Star} u) \subseteq \operatorname{Star} f(u)$ and $g(\operatorname{Star} u) \subseteq \operatorname{Star} g(u)$. Then there is an element $u \in U$ for which $f(u) \cup g(u) \subseteq \operatorname{Star} f(u)$.

Lemma B. Let $f$ and $g$ be maps of $X$ into $Y$, where $X$ and $Y$ are compact Hausdorff, $f$ is onto, and $f$ and $g$ have no incidence points. Then there exist finite coverings $U$ of $X$ and $V$ of $Y$ such that for each $u$ in $U$, there is $a v$ in $V$ for which $f(u) \subseteq v$, but $g(u) \cap \operatorname{Star}_{V} v=0$.

\section{REFERENCES}

1. S. Eilenberg and N. Steenrod, Foundations of algebraic topology, Princeton University Press, 1952.

2. O. H. Hamilton, $A$ fixed point theorem for pseudo-arcs and certain other metric continua, Proc. Amer. Math. Soc. vol. 2 (1951) pp. 173-174.

3. J. Isbell, Embeddings of inverse limits, manuscript submitted for publication to the Ann. of Math.

4. B. O'Neill and E. G. Straus, A fixed point theorem, Proc. Amer. Math. Soc. vol. 8 (1957) pp. 1148-1151.

5. W. L. Strother, On an open question concerning fixed points, Proc. Amer. Math. Soc. vol. 4 (1953) pp. 988-993.

6. L. E. Ward, Jr., A fixed point theorem, Amer. Math. Monthly vol. 65 (1958) pp. 271-272.

UNIVERSITY OF WISCONSIN 